\title{
Improving the long term outcome of schizophrenia John Csernansky*
}

Address: Department of Psychiatry, Washington University School of Medicine, St. Louis, MO, USA

* Corresponding author

\author{
from International Society on Brain and Behaviour: 2nd International Congress on Brain and Behaviour \\ Thessaloniki, Greece. 17-20 November 2005 \\ Published: 28 February 2006 \\ Annals of General Psychiatry 2006, 5(SuppI I):S28 doi:10.1 186/1744-859X-5-SI-S28
}

Relapse in schizophrenia remains common, despite its adverse consequences. Discontinuation of antipsychotic medication is a strong predictor of relapse in patients with schizophrenia. However, even when treatment with conventional antipsychotic drugs is continued, relapse still occurs in $30-50 \%$ of patients each year. The purpose of this study was to determine whether risk of relapse and residual psychopathology could be reduced and cognition and quality of life improved, by switching clinically stable subjects with schizophrenia from their usual antipsychotic drug to either risperidone (1-8 $\mathrm{mg} /$ day) or haloperidol (2.5-20 mg/day) for a minimum of one year.

Criteria for relapse were psychiatric hospitalization, need for an increase in the level of psychiatric care with a $25 \%$ increase in PANSS score, a CGI change score of 6, deliberate self-injury, suicidal/homicidal ideation or injury to others/property damage. The PANSS was used to assess residual psychopathology. Lehmann's Quality of Life Interview was used to assess quality of life and a battery of cognitive tests featuring verbal memory was used to assess cognition.

The mean time to relapse in risperidone-treated and haloperidol-treated patients was 452 days and 391 days, respectively $(p=0.001)$. From baseline to final assessment, mean total PANSS and sub-scale scores for positive symptoms, negative symptoms, disorganized thoughts, uncontrolled hostility/excitement and anxiety/depression all significantly favored risperidone over haloperidol. Treatment with risperidone was also associated with significant increases in almost all components of quality of life and cognition (verbal memory) as compared to haloperidol. Finally, adverse events of insomnia, psychosis, somnolence, agitation hyperkinesia and EPS were more frequent in the haloperidol-treated patients than in the risperidione-treated patients. Compliance was very high in both groups of patients (more than 95\% of all drug doses were taken correctly).

These results suggest that the outcome of schizoprhenia can be altered by the selection of antipsychotic drug. Specifically, risperidone as compared to haloperidol reduced the rate of relapse and residual psychopathology and improved cognition and quality of life in patients with schizophrenia at doses commonly used in clinical practice. These findings enhance the body of evidence that novel antipsychotics, such as risperidone, optimize longterm outcomes in schizophrenia. 